\title{
System design and application of sewage treatment and the use of recycled water in Dianchi Lake Basin based on WebGIS
}

\author{
Qiaoling Tang ${ }^{1}$, Hong Liang ${ }^{1}$, Yumei Guo ${ }^{2}$, Ruitao Cun ${ }^{2}$ \\ ${ }^{1}$ College of Information Science \& Engineering, Yunnan University, Kunming, China \\ ${ }^{2}$ Kunming Drainage Facilities Management Co., Ltd , Kunming, China
}

\begin{abstract}
In this paper, due to the current problem of sewage treatment in Dianchi Lake Basin in Kunming and the practical demand for the integrated solution of the sewage treatment and reclaimed water use, our team has developed the online management system based on the WebGIS. The online management system is designed based on geographic spatial information mapping technology, WebGIS technology and spatial database technology and finally displayed in the form of thematic map called "Visual information platform for Dianchi Lake Basin sewage treatment and recycling water use". The system has achieved scientific and informative management for Dianchi Lake Basin sewage treatment and recycling of water use. It also provides comprehensive and accurate basic data and decision support for the improvement of urban water environment and pollution control in Dianchi Lake in Kunming. The online system improves the management efficiency, management level and decision-making capacity of sewage treatment and recycling industries in Kunming, and provide a good demonstration and reference in infrastructure of the city.
\end{abstract}

\section{Introduction}

Water is the basic and strategic resource of social development, and it is also an important ecological environment for human beings to survive. The cities, as the major human settlements, are showing more and more dependency for the water environment and water resources. In recent years, with the rapid development of urban economic construction, the city expanding, and extensive increasing urban population, the urban water environment has deteriorated. Modern cities are facing the dual pressures of water pollution and water shortage. As a strategic hub and axis of China's southwest open bridgehead, Kunming, the water environment pollution and water shortage is particularly prominent. With the rapid development of economy in Kunming and the rapid expansion of the scale of the city, a large number of domestic sewage and industrial wastewater had been released into Dianchi Lake, resulting in water pollution which did great damage to water function and water quality.

The system in this paper is developed based on ArcGIS produced by ESRI. It is a large GIS software platform with comprehensive, complete and scalable function. In addition to the powerful processing and analysis of spatial data, the platform also provides a rich development interface including Python, Javascript, .NET and so on, which provides strong support for the research and development of this paper. At the desktop, server, browser, mobile and cloud, ArcGIS has the corresponding services and products to provide users with the complete open GIS user experience which make0 it has become the most popular geographic information system platform software.

The two main applications of this software are desktop products ArcMap and server-side products ArcGIS Server. ArcMap is mainly for implementation of data processing and other functions, ArcGIS Server is responsible for publishing good map data, a map service for later development and use. In this paper, ESRI's ArcGIS Server is used to implement Web-based map publishing technology. It uses C / S and B / S hybrid network architecture to implement programming and integration with Javascript as programming language, and realize the urban sewage treatment and resource recycling Information management platform in the research and application [1].

This system can realize the remote and dynamic visual management and analysis of sewage treatment online monitoring, sewage treatment water quality analysis data management, reclaimed water use management, and sludge disposal, and provide comprehensive and accurate sewage treatment and resource recycling information for management and decision support. (Including sewage treatment plants, sewage treatment facilities, sludge 
disposal plants, reclaimed water treatment stations, recycled water users, recycled water users, reclaimed water intake points, etc.) for the relevant management of Dianchi Lake, which is related to the management of Dianchi Lake sewage treatment and resource recycling.) Efficient storage and management of data resources, as well as data modification, editing, updating and maintenance, to ensure the real-time data, accuracy and integrity, to achieve data sharing.

\section{The establishment of database}

\subsection{Spatial data model}

In this paper, a new generation of object-oriented spatial data mode based on GeoDatabase as Dianchi Lake sewage treatment and resource recycling network information management platform for spatial data model. The model uses standard relational database technology to express the data model of geographic information, and integrates spatial data and attribute data into the standard relational database in the background to realize the seamless integration of spatial data and attribute data. It is based on RDBMS On the intelligent spatial data model.

GeoDatabase uses object-oriented technology to abstract the real world into feature classes (points, lines, faces) that contain spatial information and object classes (feature attributes) that do not contain spatial information. Each feature class and object class has attributes, behaviors, and rules that are associated with objects such as relational classes, geometric networks, etc.

\subsection{Design and Implementation of Spatial Database}

Dianchi Lake sewage treatment and resource recycling spatial database design and construction is the core of the project implementation of the basic work. Spatial database design, it is necessary to fully consider the relational database of standardized theory, the establishment of a correct reflection of the objective reality of the relationship between the reduction of data redundancy and duplication, but also taking into account the Dianchi Lake sewage treatment and resource recycling characteristics and drainage management related management Business application requirements.

As for the new generation object - oriented spatial database model - Geodatabase, the platform designs and builds a reasonable spatial data element class, object class and relation class, and designs an efficient, stable, safe and practical spatial database of urban drainage system.

Dianchi sewage treatment and resource recycling Geodatabase is in accordance with certain models and rules combined geographical elements of data sets, object classes and relational class collection to manage and use the geographic elements of data. That is, Dianchi Lake sewage treatment and resource recycling spatial database use hierarchical data objects to organize geographic data, these data objects include feature data sets (Feature Dataset), feature class (Feature Class) and object class (Object Class) [2]

In order to make the database structure clear, easy to design and development and maintenance management, Dianchi Lake sewage treatment and resource recycling spatial database use hierarchical data objects to organize geographic data, which means, the entire geographic database is divided into the following layers: basic data, sewage treatment plant (point elements), sewage treatment facilities(point elements), pollution(point elements), reclaimed water (point elements), recycled water users (point elements), reclaimed water delivery pipe network (line elements), reclaimed water intake points (point elements), as shown in Figure 1. Among them, the basic data layer for the Kunming City is the basic electronic map, including Kunming roads, neighborhoods, water systems, rivers, units, green and so on.

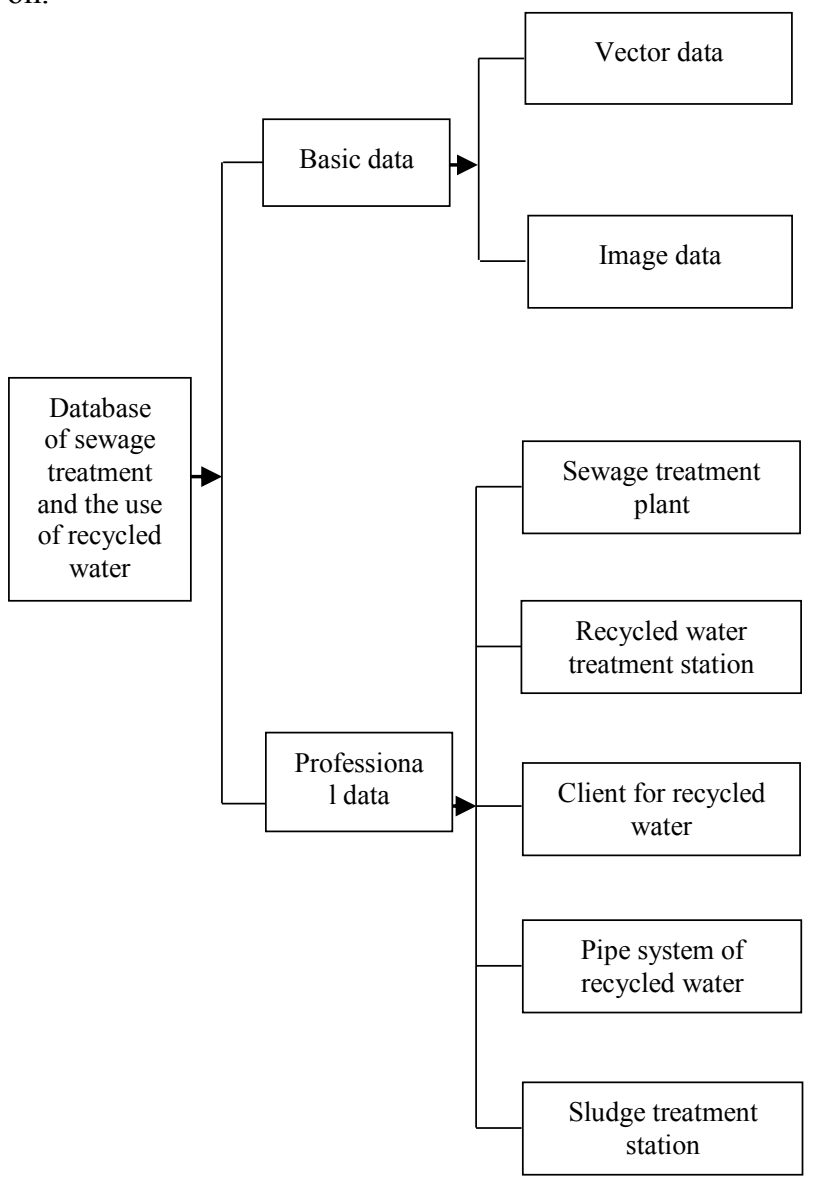

Figure 1. Spatial database layer structure

In the Dianchi Lake sewage treatment and resource recycling spatial database, the feature data set is a collection of the feature class of the shared space reference system. In the spatial database, all feature classes in the same geographic feature dataset must have the same spatial reference, which means its coordinate system, spatial domain, and the precision are the same. Spatial reference is an important part of database design. Dianchi Lake sewage treatment and resource recycling space database built in Microsoft SQL Server 2008 SDE database, achieving the creation of spatial database process through ArcCatalog. Dianchi Lake sewage 
treatment and resource recycling spatial database layer structure is shown in Figure 1.

The designing for the database of the sewage treatment and recycled water is shown in Figure 2:

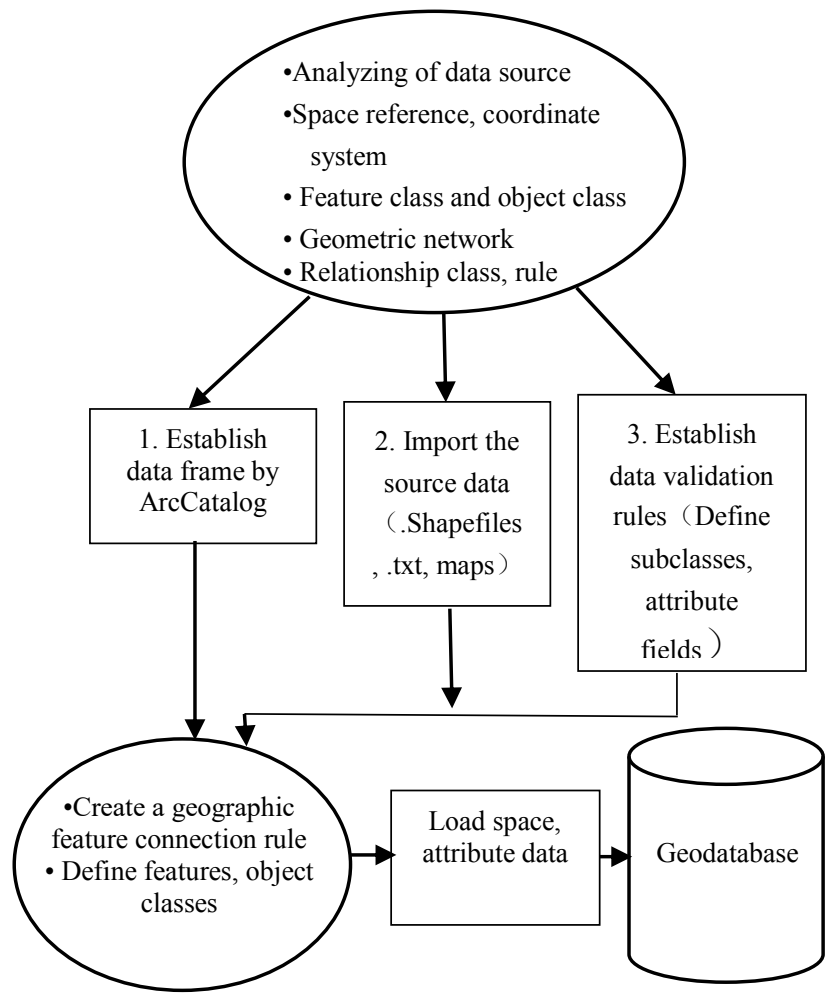

Figure 2. Designing for the database of the sewage treatment and recycled water

\section{WebGIS}

WebGIS (Web Geographic Information System) refers to the Internet-based platform using geographic information systems on the Internet, whose client application software using network protocols. WebGIS achieve spatial data sharing and interoperability through the Internet to publish and apply geospatial data, such as GIS information online query and business processing. WebGIS is a new technology to expand and perfect GIS by using Internet technology. Its core is to embed the application standard of HTTP standard in GIS, and realize spatial information management and distribution in Internet environment. WebGIS can be distributed with multiple hosts, multi-database, through the Internet / Intranet to achieve interconnection, with a browser / server (B / S) structure. The server provides information and services to the client, the browser (client) has a variety of spatial information and application functions.

WebGIS is the product of Internet technology applied to GIS development. From any node on the Web, Internet users can browse the spatial data in the WebGIS site, create thematic maps, and perform various spatial retrieval and spatial analysis.

\subsection{The structural model of WebGIS}

WebGIS has the three-tier structure with client, application server and database composition, as shown in Figure 3, the client request data services through the HTTP protocol item web server, and server return data results in web.

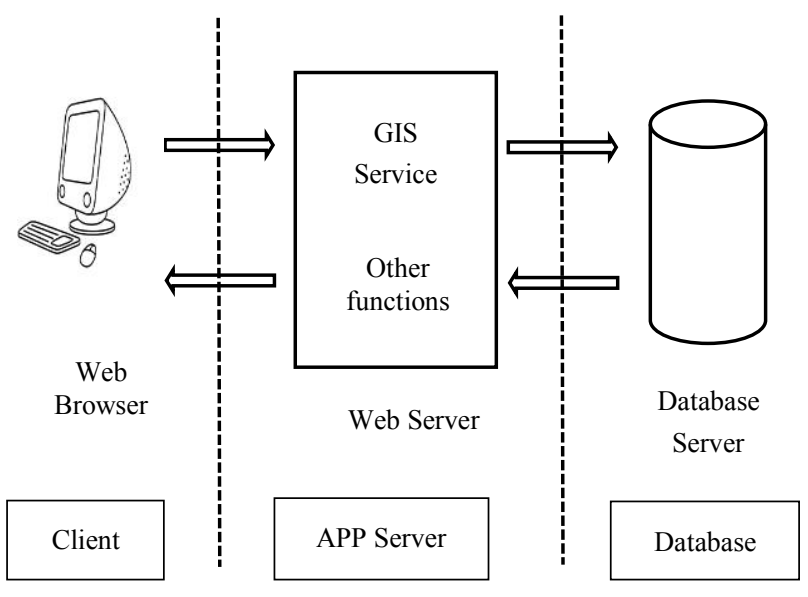

Figure 3. The structural model of WebGIS

\subsection{Design and Implementation of WebGIS}

\subsubsection{Server policy}

In this WebGIS architecture, the server finishes all the work of spatial data processing, the client is only used to issue the final result of the service request and display server, the performance requirements of the server are much higher than other situation [3].

The advantage of server policy is that the requirement for client $\mathrm{PC}$ hardware is much lower when running complex GIS operations and also can take full advantage of the existing GIS resources.

The disadvantage is that each step of the client needs sending request to the server through the network and the GIS server will also return processed results to the user in the form of static images, resulting in heavy network transmission burden. Meanwhile, the burden on the server is also heavy, and the client's operability is poor.

The architecture of the server policy is shown in Figure 4:

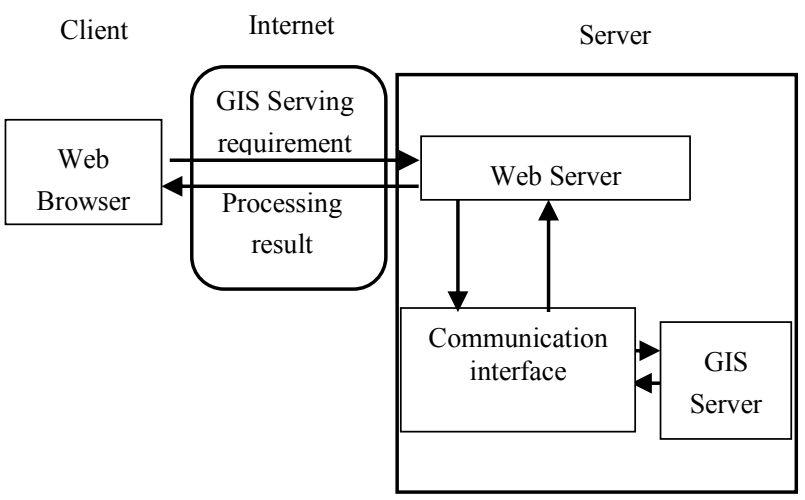

Figure 4. Architecture of the server policy 


\subsubsection{Client policy}

Client software can be built in three different technologies, namely Plug-in, ActiveX controls and Java applets [3].

The advantage for this policy is that GIS is running fast. Because all GIS operations, such as map scaling, attribute queries, etc., are done locally by the client software, so the system responds quickly. And because the server only provides GIS data services, while the server and the client do a data transmission only once, it can greatly reduce the burden of server and network transmission.

The disadvantage is that this policy use WebGIS developed based on ActiveX technology which can only be carried out under the IE browser of Microsoft's Windows; and client need to install the client plug-in before use if the WebGIS is developed based on Plug-in technology.

The architecture of the client policy is shown in Figure 5:

Client

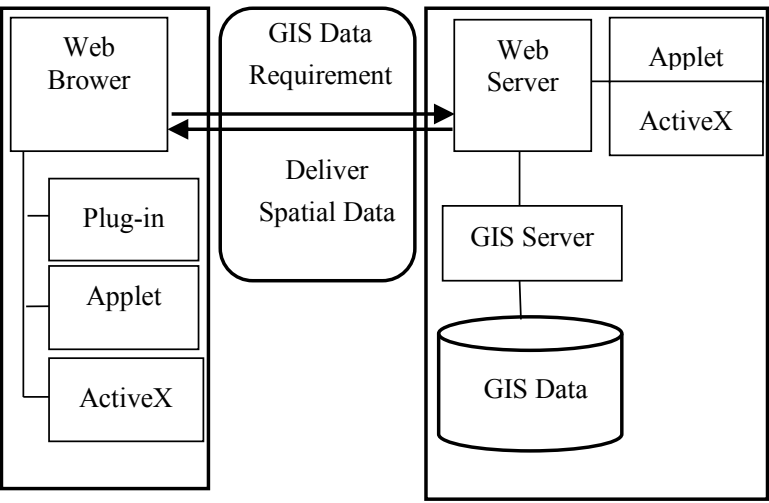

Figure 5. Architecture of client policy

\subsubsection{Mixed policy}

From the above analysis, we can see that, a simple server policy and client policy always has some limitations. When server-side policies involve frequent data transfers, their efficiency is severely affected by network bandwidth and network load. In the client policy, when the processing and processing capacity is inconsistent, by the computing power of the impact of certain tasks may run slower, or even impossible to complete [3].

So the mixed policy came into being, when it involves a lot of data operations and complex analysis tasks, you can make a strong computing power to deal with the server, when it comes to the user interaction more tasks, you can use the client to deal with, in this case, servers and clients can share their performance and data processing capabilities, thereby rationally allocating data and data processing procedures to make the overall performance of the system better.
Thematic maps, also known as special maps, are focus on one or several natural elements or socio-economic phenomenon of the map. The map area of the thematic map is broad, and information data with spatial attributes can be used to express it. The content of the thematic map consists of two parts:

1) Topic content

The natural or socioeconomic phenomenon highlighted in the figure and its related characteristics.

2) Geographic basis

To mark the thematic elements of the spatial location and geographical background of the general map content, mainly coordinate system, water, realm, residential and so on.

The use of thematic maps can study the distribution characteristics and laws of various thematic phenomena, can also study the interrelationships and dynamic changes of various phenomena., predict the coming trends and changes, do the comprehensive evaluation, zoning and planning.

\subsection{Method of Realizing Feature Map Function}

WebGIS implementation of the thematic map function can be achieved using the following methods: XSLT technology, XSLT used to convert an XML to other structures of the XML, the XSLT processor use XSLT style form of the application to the XML document to output XHTML, SVG or other documents in XML format. Since the SVG file conforms to the XML standard, you can write a good XSL style sheet file according to the different requirements, and then apply the style sheet to the original map SVG file to generate a new SVG file containing the thematic map to the user. Another way is that, because the SVG file is also an XML file, according to the user's thematic map needs, directly to the file analysis and modification, and then modify and save the map SVG file back to the user. The third method, you can write the Javascript code for the SVG file document object model that DOM operation to achieve the thematic map function, this article uses this method to achieve the thematic map function [4].

\subsection{The realization of thematic map function}

The thematic map is an important function of GIS, which can intuitively express one or more data related to the topic, making the data more convincing. The creation of thematic maps is generally divided into two ways: one way is to change the existing layer, for example, change the color of each region in a layer, with different colors on behalf of the size of different data; another way is based on the existing layer to add some new layers, these layers include some dots, circles and other graphic symbols, with these graphical symbols to the image of the amount of data[5]. This article uses the second approach. Figure 6 is a thematic map showing all the pump stations in circles.

\section{Thematic map}




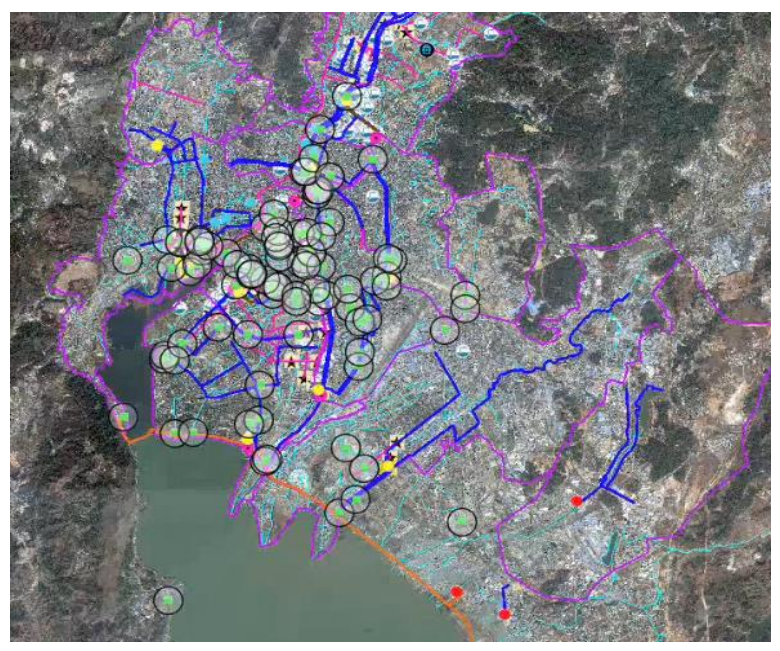

Figure 6. Thematic map

\section{Conclusions}

The WebGIS system allows people to easily browse or retrieve a variety of distributed geospatial data on the web from any node on the internet and conduct a variety of on-line geospatial analysis. The emergence of the WebGIS system enables GIS to truly move towards society and serve social groups. The design and application of WebGIS in Dianchi Lake Basin has realized the scientific and informative management of wastewater treatment and reclaimed water in Dianchi Lake Basin, and provided comprehensive and accurate basic data and scientific decision for Kunming water environment improvement and Dianchi Lake pollution control at the same time.

\section{References}

1. Huang, Shao Hua, and R. T. Huang. "Analysis and Design of Wuhan Tourism Information System Based on WebGIS." Bulletin of Surveying \& Mapping (2003).

2. Choi, Jinmu, W. Cooke, and M. Stevens. "Designing a Geodatabase for Fire Risk Assessment.".

3. Zhou, Kun, et al. "RESEARCH AND APPLICATION OF WEBGIS BASED ON SVG TECHNOLOGY." Contributions to Geology \& Mineral Resources Research 21.1(2006):49-53.

4. Zhang, Yihan. "DESIGN AND DEVELOPMENT OF ENVIRONMENTAL MONITORING DATA MANAGEMENT SYSTEM BASED ON WEBGIS."Computer Applications \& Software (2007).

5. Lin, Shouyi, et al. "Design and Implementation of Lujiazui Land Management Information System Based on WebGIS." International Conference on E-Business and E-Government IEEE Computer Society, 2010:667-670. 